\title{
In situ formation of graphene/metal oxide composites for high-energy microsupercapacitors
}

\author{
Jaemin Jung', Jae Ryeol Jeong', Jungjun Lee', Sang Hwa Lee', Soo Young Kim $\mathbb{D}^{2}$, Myung Jun Kim', \\ Junghyo Nah $\mathbb{B}^{3}$ and Min Hyung Lee $\mathbb{B}^{1}$
}

\begin{abstract}
The current design trends in the field of electronic devices involve efforts to make these devices smaller, thinner, lighter, and more flexible. The development of such systems is expected to further accelerate, resulting in the production of wearable and Internet-of-Things devices. In this respect, microenergy storage systems with high capacity and fast charge/discharge rates have become important power sources for such devices. In particular, interdigitated microsupercapacitors (MSCs) have exhibited remarkable potential as micropower sources owing to their fast charge/discharge processes, long cycle life, and high power density compared with microbatteries. Nevertheless, facile fabrication of MSCs using interdigitated electrodes remains challenging, as it requires selective decoration of electrodes with pseudocapacitive materials, such as transition metal oxides, to increase their capacitance. In the present study, we developed a simple method for fabricating MSCs involving in situ formation of interdigitated graphene electrodes and $\mathrm{ZnO}$ nanorods by photothermal conversion of graphene oxide (GO) and Zn precursors using infrared (IR) laser scribing. The fabricated MSCs exhibit a high stack capacitance of $3.90 \mathrm{~F} \mathrm{~cm}^{-3}$ and an energy density of $0.43 \mathrm{mWh} \mathrm{cm}^{-3}$. Notably, the capacity of the developed material is three times higher than those of previously reported MSCs made from the same type of graphene. In addition, the capacitance retention rate of the fabricated MSC is approximately $70 \%$ when measured over 10,000 charging-discharging cycles at a constant current, which evidently indicates a stable device performance.
\end{abstract}

\section{Introduction}

The recent research trends in electrochemical storage systems have mainly focused on manufacturing light, thin, compact, and flexible units suitable for portable smart electronic devices while enhancing their performance and efficiency. However, such efforts are often associated with challenges, including short circuits during miniaturization and compatibility issues for new device architectures with conventional electrode materials. To alleviate these problems, considerable efforts have been made to fabricate

\footnotetext{
Correspondence: Junghyo Nah (jnah@cnu.ac.kr) or

Min Hyung Lee (minhlee@khu.ac.kr)

${ }^{1}$ Department of Applied Chemistry, Kyung Hee University, Yongin, Gyeonggi

17104, Korea

${ }^{2}$ Department of Materials Science and Engineering, Korea University, Seoul

02841, Korea

Full list of author information is available at the end of the article.

These authors contributed equally: Jaemin Jung, Jae Ryeol Jeong
}

microsupercapacitors (MSCs) exhibiting high energy and power densities utilizing various micropatterning methods, such as lithography techniques ${ }^{1}$, selective wettinginduced patterning ${ }^{2}$, and laser-induced patterning ${ }^{3,4}$. Nevertheless, the limited enhancement in the capacitor performance has been demonstrated to be a consequence of the inherently low energy density of the electric double layers formed by the carbon materials used for the fabrication of most devices. Thus, it is essential to develop a redox-active system able to increase the energy density (i.e., by employing conducting polymers and metal oxides) utilizing the Faradaic reaction and to establish appropriate micropatterning methods.

Herein, we report a simple and reliable laser-induced $\mathrm{ZnO}$ nanorod (NR)/reduced graphene oxide ( $\mathrm{rGO}$ ) patterning technique for fabricating solid-state planar MSCs that exhibit both electric double-layer capacitance and 
pseudocapacitance. We used imaging software to design various electrodes based on $\mathrm{ZnO} \mathrm{NRs} / \mathrm{rGO}$, and selective laser irradiation was conducted using a general digital versatile disk (DVD) recording drive. For the development of optimal MSCs, the capacitive performances of three different designs of $\mathrm{ZnO} \mathrm{NR} / \mathrm{rGO}$ MSCs were compared. Our results demonstrate that the $\mathrm{ZnO} \mathrm{NR} / \mathrm{rGO}$ MSCs retain a stack capacitance and an energy density of $3.90 \mathrm{~F}$ $\mathrm{cm}^{-3}$ and $0.43 \mathrm{mWh} \mathrm{cm}^{-3}$, respectively, which are approximately threefold higher than the values of the bare rGO MSC. Despite the marginally lower conductivity of our laser-induced rGO compared with a previous study ${ }^{3}$, the $\mathrm{ZnO} \mathrm{NR} / \mathrm{rGO}$ MSCs exhibit a notable performance improvement. The method described in the current study necessitates a redox reaction and a high electrical conductivity. Concurrently, it is remarkably simple and applicable to other electrochemical microdevices.

\section{Materials and methods}

A polyethylene terephthalate (PET) film was cut into a disk shape to fit on a LightScribe label DVD. In this process, the PET film was carefully loaded onto the DVD to avoid covering the unique codes at the center of the DVD, which were used for precise positioning of the disc. Notably, the codes were useful for repeating laser scanning at exactly the same positions to achieve further reduction of GO. An aqueous dispersion of GO with a concentration of $2.0 \mathrm{mg} \mathrm{mL}^{-1}$ was prepared via a modified Hummers method. Subsequently, $20 \mathrm{~mL}$ of the GO solution was drop-cast on the prepared PET film and dried overnight to obtain a homogeneous GO-coated PET film. A $5 \mathrm{~mm}$ solution of zinc acetate dehydrate (MW 219.50, 99.0\%, Samchun Chemicals, Korea) in ethanol was prepared as the zinc ion precursor. The as-prepared GO film was then wetted with the zinc acetate precursor solution and rinsed with ethanol after $30 \mathrm{~s}$. These steps were repeated five times to increase the density of the precursors on the GO film. Finally, the zinc acetatetreated GO film was scanned along the interdigitated electrode design using the infrared (IR) laser in a LightScribe DVD drive (HP-337 s). These steps were repeated three times to simultaneously obtain highly reduced GO and $\mathrm{ZnO}$ seeds. Importantly, to form high-density $\mathrm{ZnO}$ seeds, the zinc acetate wetting step and the laser scanning process were repeated.

\section{Growth of ZnO NRs based on $\mathrm{ZnO}$ seeds/rGO}

The zinc precursor solution for $\mathrm{ZnO}$ growth was prepared by dissolving $2.97 \mathrm{~g}$ of zinc nitrate hexahydrate (MW 297.47, 98.0\%, Samchun Chemicals, Korea) and $1.40 \mathrm{~g}$ of hexamethylenetetramine (MW 140.19, 99.0\%, Kanto-Chemical, Japan) in $400 \mathrm{~mL}$ of deionized water. Subsequently, the as-prepared $\mathrm{ZnO}$ seed/rGO film was placed upside down on the surface of the zinc precursor solution, and the hydrothermal reaction was maintained at $90^{\circ} \mathrm{C}$ for $6 \mathrm{~h}$. Following the completion of the reaction, the $\mathrm{ZnO} \mathrm{NR} / \mathrm{rGO}$ film was immediately washed with deionized water and dried under ambient conditions.

\section{Preparation of the $\mathrm{H}_{2} \mathrm{SO}_{4} / \mathrm{PVA}$ gel electrolyte}

Approximately $1 \mathrm{~g}$ of polyvinyl alcohol (PVA; MW 89,000-98,000, $99+\%$ hydrolyzed, Sigma-Aldrich, USA) and $10 \mathrm{~mL}$ of deionized water were mixed, and the mixture was stirred at $90^{\circ} \mathrm{C}$ until the solution turned transparent. The PVA solution was subsequently cooled to room temperature prior to the addition of $1 \mathrm{~g}$ of $\mathrm{H}_{2} \mathrm{SO}_{4}$ (MW 98.07, 95.07\%, Samchun Chemicals, Korea). The reaction mixture was stirred until a homogeneous gel solution was obtained.

\section{Fabrication of $\mathrm{ZnO} \mathrm{NR} / \mathrm{rGO}$ MSCs and the rGO MSC}

Laser-induced rGO served not only as electrodes but also as current collectors. The as-prepared $\mathrm{H}_{2} \mathrm{SO}_{4} / \mathrm{PVA}$ gel electrolyte was drop-cast on the interdigitated finger electrode area of the MSCs. The excess solvent was then allowed to evaporate overnight under ambient conditions to form a solid electrolyte film on the MSCs. Subsequently, the current collector area was coated with a silver paste (Elcoat p-100, CANS) to protect the rGO contact pads from scratching by the electrochemical workstation probes.

\section{Material characterization}

All synthesized materials were characterized by field emission scanning electron microscopy (FESEM; LEO SUPRA 55), high-resolution transmission electron microscopy (HRTEM; JEOL JEM-2100F, 200 kV), X-ray diffraction (XRD; D8 Advance equipped with a $\mathrm{Cu} \mathrm{K}_{\alpha}$ source), and X-ray photoelectron spectroscopy (XPS; Thermo Electron K-Alpha). All XPS spectra were calibrated to the binding energy of the $\mathrm{C} 1 \mathrm{~s}$ peak at $284.6 \mathrm{eV}$.

\section{Electrochemical measurements}

The capacitive performance of the obtained MSCs was evaluated utilizing various electrochemical analysis techniques, including cyclic voltammetry $(\mathrm{CV})$, galvanostatic charge-discharge (GCD) tests, electrochemical impedance spectroscopy (EIS), and GCD cycling stability tests. The CV, GCD, and EIS measurements were performed using a potentiostat/galvanostat (IVIUMSTAT, Ivium Technologies). The EIS measurements were recorded over a frequency range of $100 \mathrm{kHz}$ to $0.01 \mathrm{~Hz}$ with an amplitude of $10 \mathrm{mV}$ at the open-circuit potential. The GCD cycling stability test was conducted using a multichannel potentiostat/galvanostat (WMPG1000, WonATech) at a $0.1 \mathrm{~A} \mathrm{~cm}^{-3}$ current density. 


\section{Results and discussion}

\section{Fabrication of ZnO NR/rGO MSCs}

The fabrication process of the $\mathrm{ZnO} \mathrm{NR} / \mathrm{rGO}$ patterns is summarized in Fig. 1. Graphene oxide was synthesized using the modified Hummers $\operatorname{method}^{5}$. First, the GO solution was drop-cast on a PET film and dried overnight. Subsequently, the as-prepared GO film was wetted with the zinc acetate precursor solution and rinsed with ethanol after $30 \mathrm{~s}$. The wetting and rinsing steps were repeated five times to obtain highly homogeneous and concentrated zinc acetate embedment on GO. The designated area of the GO with the zinc acetate film was scanned three times using the DVD $783 \pm 3 \mathrm{~nm}$ IR laser. During this step, a high surface-to-volume ratio of GO was photothermally deoxygenated (Supplementary Fig. $\mathrm{S} 1)^{3,6,7}$. It is challenging to dissipate the absorbed heat in nanomaterials, such as GO; therefore, the local temperature of GO rapidly increased up to nearly $1000^{\circ} \mathrm{C}$ upon exposure to the IR laser ${ }^{7}$. Consequently, GO was reduced by laser scanning, and zinc acetate was thermally decomposed to $\mathrm{ZnO}$ seeds as a result of the local heating $^{8-11}$. At the end of this process, $\mathrm{ZnO}$ seed/rGO complexes with the electrodes were formed, followed by hydrothermal growth of epitaxial $\mathrm{ZnO} \mathrm{NRs}$ on the $\mathrm{ZnO}$ seeds in the zinc precursor solution at $90^{\circ} \mathrm{C}$ for $3 \mathrm{~h}$ and $6 \mathrm{~h}$ to obtain line and plane patterns, respectively ${ }^{8,12}$. Thus, $\mathrm{ZnO} \mathrm{NR} / \mathrm{rGO}$ composites were successfully formed at the laser-scribed locations and could be used for electrochemical applications.

Notably, when the laser scanned over the GO film, the morphology of the exposed GO obviously changed via rapid thermal deoxygenation. The rapid heating process exfoliated and reduced the GO sheets by decomposing the oxygen-containing moieties, resulting in swollen rGO morphologies (Supplementary Fig. S2). The concurrent decomposition of the oxygen groups also removed carbon atoms from the carbon lattice, inducing distortion of the carbon plane of $\mathrm{rGO}^{13}$. This change is particularly evident in the central part of the laser scanning line, where the irradiated laser power density is higher than that at the edges of the laser spot; thus, cracks are often observed (Fig. 2). Accordingly, the growth of $\mathrm{ZnO} \mathrm{NRs}$ on the cracks (middle line) of the laser-scanned rGO was inhibited since $\mathrm{ZnO}$ seeds rarely exist on such drastically deformed structures.

Supplementary Fig. S3 presents top-view scanning electron microscopy (SEM) images of the $\mathrm{ZnO} \mathrm{NR} / \mathrm{rGO}$ line patterns. Since the resolution of the IR laser in the DVD writer is approximately $20 \mu \mathrm{m}, \mathrm{ZnO} \mathrm{NR} / \mathrm{rGO}$ line arrays with approximately $20 \mu \mathrm{m}$ line width and spacing were fabricated. As presented in Supplementary Fig. S3, selectively grown $\mathrm{ZnO} \mathrm{NRs}$ are observed along the rGO lines, especially on the edges of the rGO lines (Fig. 2). Although the unexposed zones are located directly next to the laser-scribed zones, the material remains as a flat GO film without any noticeable $\mathrm{ZnO} \mathrm{NR}$ growth, indicating the low heat conductivity of $\mathrm{GO}^{14}$.

With a view to applying the laser patterning technique in the field of electrochemical devices, we demonstrated patterning of an MSC with interdigitated negative and positive electrodes (Fig. 3). Overall, interdigitated patterns between two rectangular electrical contact pads were obtained, confirming that laser scribing can be utilized for forming both simple and complex device patterns. The length, width, and interval of each finger electrode in the interdigitated pattern are $5 \mathrm{~mm}, 300 \mu \mathrm{m}$, and $100 \mu \mathrm{m}$, respectively. Moreover, the size of the square contact pads is approximately $8 \times 8 \mathrm{~mm}$. First, we investigated the square plane of the $\mathrm{ZnO}$ seed/rGO sample prior to the hydrothermal growth of $\mathrm{ZnO}$ NRs. Figure 3b shows that the area is filled with $20 \mu \mathrm{m}$ lines of swollen rGO, which correspond to the tracks of the laser scan. Furthermore,

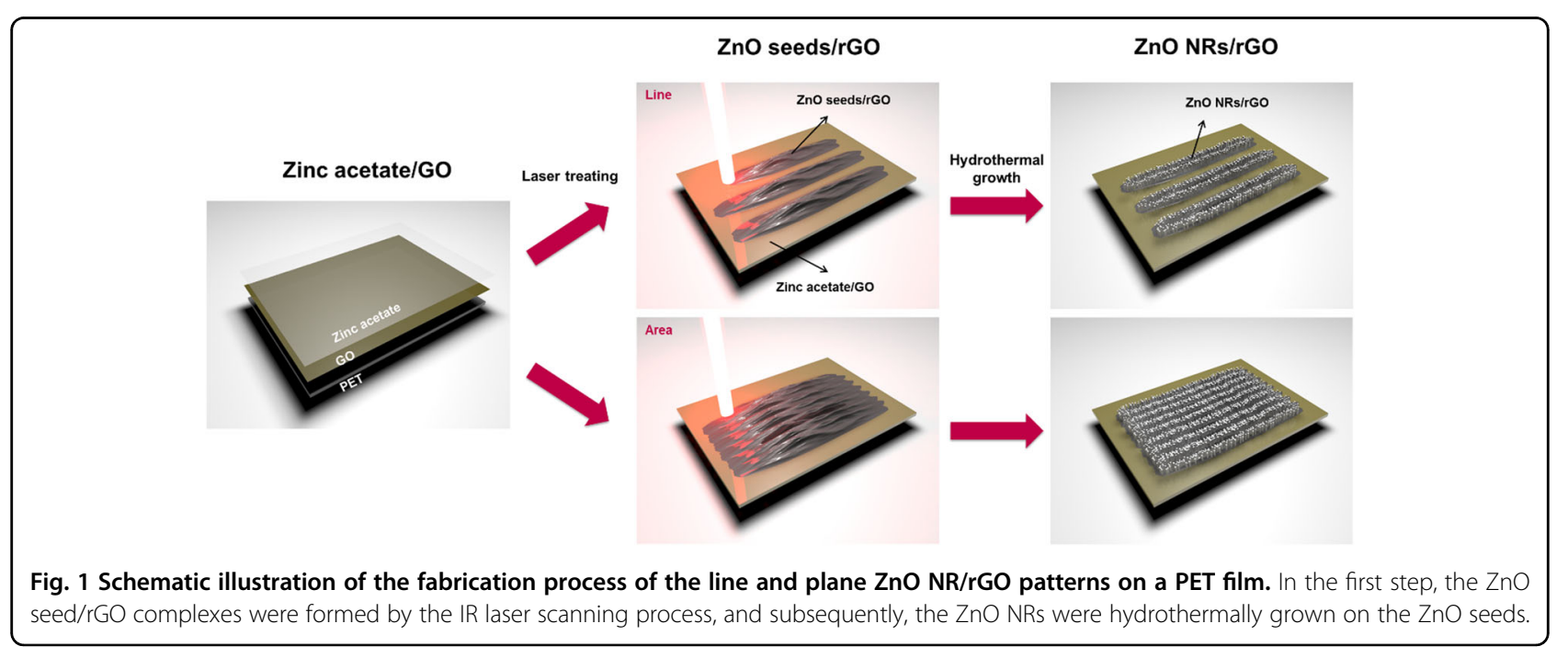




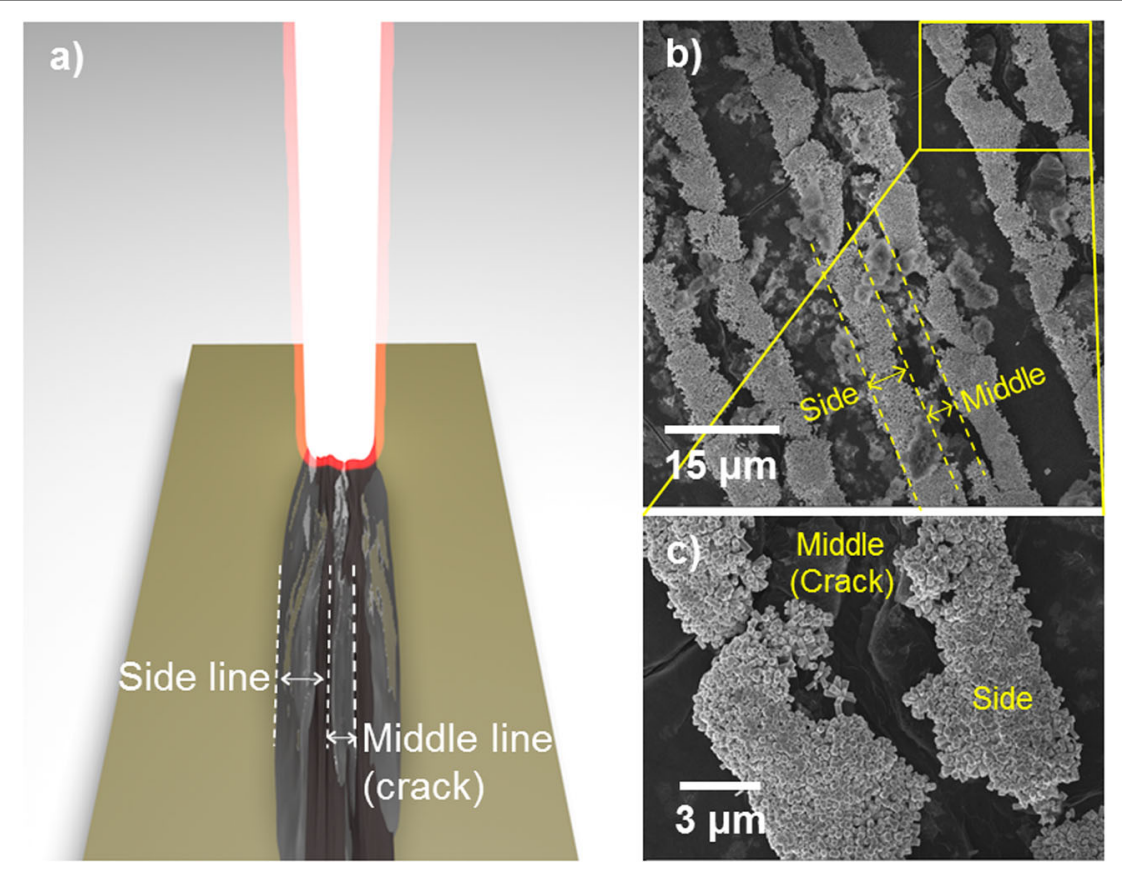

Fig. 2 Microscopic analysis of laser-scribed ZnO NRs/rGO. a Schematic illustration of laser-scanned rGO. The scanned region is divided into two distinct areas: middle line and side line. The middle line is formed as a result of rapid photothermal deoxygenation. $\mathbf{b}$, c Scanning electron microscopy (SEM) images of ZnO NRs/rGO demonstrating the preferential growth of ZnO on the side line of the swollen laser-scanned $r G O$.

the $\mathrm{ZnO}$ seed layer on $\mathrm{rGO}$ formed by thermal decomposition of zinc acetate is too thin to be clearly observed in the SEM images. The interdigitated region is also filled with rGO lines, and an unexposed GO gap region between the interdigitated electrodes is evident (Fig. 3c). Following the hydrothermal growth of the $\mathrm{ZnO}$ seed/rGO complexes in the $\mathrm{ZnO}$ precursor solution, a high density of $\mathrm{ZnO}$ NRs is observed on the rGO surface in the places where the formation of $\mathrm{ZnO}$ seeds was expected, revealing that the $\mathrm{ZnO}$ seeds on the laser-scribed $\mathrm{rGO}$ regions were nucleation sites for $\mathrm{ZnO}$ NRs (Fig. 3e-f). As presented in Fig. 3g, the white $\mathrm{ZnO}$ NR lines are composed of urchinshaped $\mathrm{ZnO}$ NRs with clearly visible groups of $\mathrm{ZnO}$ NRs ( $4 \mu \mathrm{m}$ in length and diameters of $70-120 \mathrm{~nm}$ ). The crystallinity of the grown $\mathrm{ZnO}$ NRs was characterized utilizing selected area electron diffraction (SAED) analysis. The evaluation confirmed that the $\mathrm{ZnO}$ NRs exhibit a hexagonal wurtzite phase that grew along the $c$-axis (Fig. S4). It is noteworthy that the proposed laser-scribed patterning methodology can be adopted to generate more complex device patterns (Fig. S5).

\section{Material characterization and evaluation of the growth mechanism of $\mathrm{ZnO} \mathrm{NRs} / \mathrm{rGO}$}

To further investigate the selective growth mechanism of the ZnO NRs on swollen rGO, the XRD and XPS spectra collected from both the IR laser-unscanned (GO) and laser-scanned ( $\mathrm{ZnO} \mathrm{NRs} / \mathrm{rGO})$ zones were analyzed
(Supplementary Fig. S6). Three distinct peaks at $31.9^{\circ}$, $34.6^{\circ}$, and $36.4^{\circ}$ are observed in the XRD spectra, which correspond to (1010), (0002), and (1011) of wurtzite $\mathrm{ZnO}$, respectively (Supplementary Fig. S6a). For the laserscanned zone, the presence of the sharp and strong peak at $34.6^{\circ}$ confirms that the synthesized $\mathrm{ZnO}$ NRs primarily grew along the $c$-axis ${ }^{15}$. In contrast, there are no peaks corresponding to the $\mathrm{ZnO} \mathrm{NRs}$ in the unscanned zone. This result suggests that the $\mathrm{ZnO}$ NRs can only be grown in the laser-scanned zone. Moreover, in both XRD patterns, there are no specific peaks corresponding to $\mathrm{GO}$ or $\mathrm{rGO}$, as the PET substrate is considerably thicker than the GO and rGO films. To further investigate the formation of $\mathrm{ZnO} \mathrm{NRs} / \mathrm{rGO}$, XPS analysis was conducted after each step (Supplementary Fig. S6). First, C 1 s XPS evaluation was performed in the laser-scanned zone (rGO) and the laser-unscanned zone (GO) to confirm the photothermal reduction of GO under IR laser scribing (Supplementary Fig. S6b-c). Distinctive GO peaks, including $\mathrm{C}-\mathrm{O}(286.3 \mathrm{eV}), \mathrm{C}=\mathrm{O}(288.1 \mathrm{eV}), \mathrm{O}-\mathrm{C}=\mathrm{O}$ $(289.4 \mathrm{eV})$, and $\mathrm{C}-\mathrm{C} / \mathrm{C}=\mathrm{C}(284.6 \mathrm{eV})$, are observed in the GO area (Supplementary Fig. S6b); however, the first three oxygenated carbon peaks nearly disappear following the laser scanning treatment in the rGO areas (Supplementary Fig. S6c). Furthermore, the Raman spectra were also investigated to evaluate the quality of rGO depending on the laser-scribing time (Supplementary Fig. S7). Two distinctive peaks are observed at approximately 

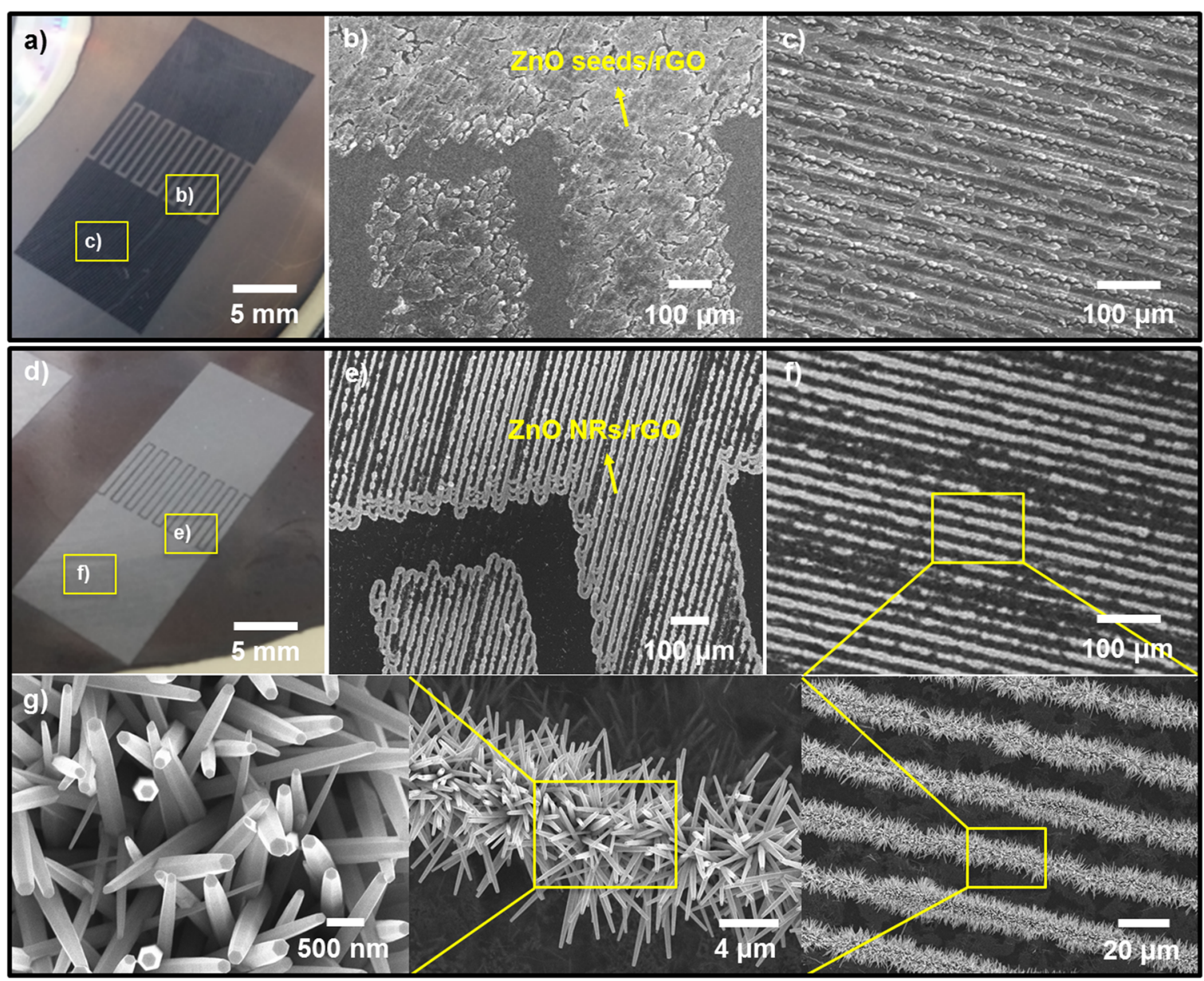

Fig. 3 Laser scribing of ZnO NR/rGO MSCs. a Photograph of the MSC electrode patterns (black) with ZnO seeds/rGO following laser scribing. b, c Magnified SEM images of the marked areas in a. d Photograph of the $\mathrm{ZnO} N R / \mathrm{rGO}$ electrode patterns after 6 h of hydrothermal growth of the $\mathrm{ZnO}$ seed/rGO electrodes in zinc precursor solutions. The photograph shows that the color changed from black to gray with the growth of the $\mathrm{ZnO}$ NRs. e, $\mathbf{f}$ Magnified SEM images of ZnO NRs/rGO from the marked regions in $\mathbf{d}$. $\mathbf{g}$ High-resolution SEM images of $\mathbf{f}$. The ZnO NRs clearly exhibit the hexagonal wurtzite phase.

$1350 \mathrm{~cm}^{-1}$ and $1580 \mathrm{~cm}^{-1}$, corresponding to the D band and $\mathrm{G}$ band, respectively. As the laser-scribing time is increased, the intensity ratio of $\mathrm{D}$ and $\mathrm{G}$ bands $\left(I_{\mathrm{D}} / I_{\mathrm{G}}\right)$ gradually decreases from 0.90 to 0.57 for the pristine GO film and for the tenfold laser-scribed rGO, respectively. Additionally, the $2 \mathrm{D}$ peak at $2700 \mathrm{~cm}^{-1}$ increases, confirming the graphitic nature of the films containing fewer defect sites with increasing laser-scribing time ${ }^{16,17}$. Subsequently, the Zn 2p XPS spectra were analyzed to confirm the formation of $\mathrm{ZnO}$ seeds and $\mathrm{ZnO} \mathrm{NRs}$ on rGO (Supplementary Fig. S6d, e). Supplementary Fig. S6d presents the Zn 2p XPS spectrum in the GO area (i.e., area not exposed to the IR laser) of $\mathrm{ZnO}$ seeds/rGO and $\mathrm{ZnO}$ NRs/rGO before and after hydrothermal growth, respectively. The two $\mathrm{Zn} 2 \mathrm{p}$ peaks that are clearly visible in the $\mathrm{GO}$ area correspond to the $\mathrm{Zn} 2 \mathrm{p}_{1 / 2}$ and $\mathrm{Zn} 2 \mathrm{p}_{3 / 2}$ binding energies. Thus, some zinc atoms from zinc acetate remained on GO even after rinsing with deionized water.
Compared with the Zn 2p XPS spectrum in the GO area, the $\mathrm{Zn} 2 \mathrm{p}_{3 / 2}$ peak shifts $\sim 0.4 \mathrm{eV}$ toward a higher binding energy in the rGO area, which is inconsistent with the downshifted peaks observed for the previously reported formation of $\mathrm{ZnO}$ by thermal decomposition of zinc acetate (Supplementary Fig. S6e) ${ }^{18,19}$. The noted upshift indicates an interaction of the divalent zinc ions with the oxygen functional groups of GO (Supplementary Fig. S8). Actually, divalent metal ions prefer to bind to oxygen atoms on the surface of GO, and as a result, the ions often induce cross-linking of the GO sheets ${ }^{20}$. Compared with the bare GO film (Supplementary Fig. S9a), the intensity of the $\mathrm{C}-\mathrm{O}$ bond is much lower in the zinc acetate-treated GO film, i.e., in the presence of zinc ions, indicating the influence of the divalent metal ions on the structure of the GO film (Supplementary Fig. S6b) ${ }^{20}$. Thus, the zinc ions bound to the surface oxygen atoms of the GO film and were converted to $\mathrm{ZnO}$ seeds following the laser 
treatment. In addition, the $\mathrm{Zn}$ XPS spectrum of the $\mathrm{ZnO}$ $\mathrm{NR} / \mathrm{rGO}$ area exhibits a nearly identical peak position $\left(1022.5 \mathrm{eV}\right.$ of $\left.\mathrm{Zn} 2 \mathrm{p}_{3 / 2}\right)$ to that observed in the spectrum of the $\mathrm{ZnO}$ seed/rGO area $\left(1022.4 \mathrm{eV}\right.$ of $\left.\mathrm{Zn} 2 \mathrm{p}_{3 / 2}\right)$; however, a much higher intensity of the $\mathrm{Zn} 2 \mathrm{p}$ peaks is noted in this case. Based on the analysis of the XPS peaks, we conclude that the $\mathrm{ZnO}$ NRs were successfully grown on the $\mathrm{ZnO}$ seeds, which selectively changed from divalent zinc ions on the $\mathrm{GO}$ sheets.

\section{Electrochemical characterization of the ZnO NR/rGO MSCs}

One of the key advantages of the developed patterning technique is that it provides flexibility in the design of supercapacitor electrodes using computer software. Various designs of $\mathrm{ZnO} \mathrm{NR} / \mathrm{rGO}$ microsupercapacitors (ZG MSCs) can be patterned on a PET film, and the optimum MSC design can be determined by evaluating the performance of each MSC. In the present study, three different ZG MSCs containing 14 electrodes with widths of 350,330 , or $310 \mu \mathrm{m}$ were fabricated. Notably, the same device area was retained for all MSCs (Supplementary Fig. S10). The electrochemical characterization of the developed MSCs was performed using a two-electrode system containing a solid $\mathrm{H}_{2} \mathrm{SO}_{4} / \mathrm{PVA}$ electrolyte. Figure $4 \mathrm{a}$ presents the CV curves of the 350-, 330-, and 310-ZG MSCs at a scan rate of $100 \mathrm{mV} \mathrm{s}^{-1}$. The average integral area of the CV curves of the 350-ZG MSC is considerably larger than those of the 330-ZG MSC and 310-ZG MSC, suggesting a larger stack capacitance of the 350-ZG MSC. The electrochemical performance of the ZG MSC samples with different electrode sizes was further studied by galvanostatic charge-discharge (GCD) measurements. The typical GCD test of each ZG MSC was conducted at a current density of $0.1 \mathrm{~A} \mathrm{~cm}^{-3}$ (Fig. 4b). The GCD curve of the $350-$ ZG MSC is considerably prolonged and exhibits a lower $I R$ drop in comparison to the other ZG MSCs. The stack capacitances of each ZG MSC were calculated utilizing the discharge curves and found to be equal to 3.90 , 2.32 , and $1.51 \mathrm{~F} \mathrm{~cm}^{-3}$ for the 350-, 330-, and 310-ZG MSCs, respectively. Because evaluation of the capacitance based on volume rather than mass is more reasonable in the case of MSCs, we determined the specific capacitances of the MSCs based on the volume of the stack ${ }^{3}$. Since the electrodes of the MSCs were not passivated, the Coulombic efficiencies of all ZG MSCs were higher than 100\% due to the possible interactions between the electrolytes and the $\mathrm{ZnO}$ nanorods (Supplementary Table S1). Thus, further studies are necessary to suppress undesired chemical reactions that induce breakdown of the electrodes and dissolution of active materials through the introduction of proper passivation layers ${ }^{21}$. Fig. $4 \mathrm{c}$ presents the Nyquist plots of the ZG MSCs over the frequency range of $100 \mathrm{kHz}$ to $0.01 \mathrm{~Hz}$ measured at the open-circuit potential. As shown in Supplementary Fig. S11, we created an equivalent circuit for fitting the experimental data since there is no simple equivalent circuit for porous electrodes that can be applied to our electrodes because of the complicated 3D geometry and porous structures. $R_{1}$ in the equivalent circuit corresponds to the equivalent series resistance (ESR) that includes the intrinsic resistance of the electrode material, solution resistance, and contact resistance at the interface between the current collector and electrode ${ }^{22-24}$. There are two resistor and constant phase element (CPE) pairs connected in series with $\mathrm{R}_{1}{ }^{25}$. One is related to the charge transfer process occurring at the interface between $\mathrm{ZnO}$ and the electrolyte ${ }^{22}$, and the other with Warburg impedance could be due to a charge transfer process affected by ion transport within the porous $\mathrm{ZnO}$ electrode and ion/electron hopping in the $\mathrm{ZnO}$ electrodes ${ }^{22,26}$. By fitting the experimental data to the equivalent circuit, it is found that the total resistance and Warburg resistance increase in the order of the 350-, 330-, and 310-ZG MSC (Supplementary Fig. S11b). This trend is the reverse of the order of the capacitance values, which are measured as decreasing in the order of the 350-, 330-, and 310-ZG MSC. Compared to the other ZG MSCs, the 350-ZG MSC exhibits the lowest resistance and Warburg diffusion impedance as the gap between the electrodes decreases ${ }^{27}$. The decrease in the interspace reduces the ion diffusion length and lowers the internal resistance. The stack capacitances of the different ZG MSCs as a function of the current density are presented in Fig. 4d. The stack capacitances of all the ZG MSCs decrease with increasing current density; however, the 350-ZG MSC exhibits the highest stack capacitance among all the ZG MSCs. All of the observed trends originate from the increased interspacing between the electrodes resulting from the narrowing of the electrode width in the sequence of the 350-, 330-, and 310-ZG MSC. Thus, as mentioned above, the increase in the interspace between the electrodes leads to an increase in the ion diffusion length, resulting in an increase in the internal resistance ${ }^{2}$. These observations correlate with the EIS (Fig. 4c) and $I R$-drop (Fig. 4b) results. In addition, the stack capacitance also decreases with the reduction in the electrode width.

To verify the pseudocapacitive contribution of the $\mathrm{ZnO}$ NRs in the ZG MSCs, we conducted an electrochemical performance comparison with a ZnO-free 350-rGO MSC exhibiting only electric double-layer capacitance (EDLC). The CV curve of the $350-\mathrm{rGO}$ MSC at a $100 \mathrm{mV} \mathrm{s}^{-1}$ scan rate exhibits a more rectangular shape than that of the 350-ZG MSC (Supplementary Fig. S12a). In addition, the ZG MSC exhibits a larger IR drop than the rGO MSC at the same current density (Supplementary Fig. S12b). In other words, in contrast to the rGO MSC, the ZG MSC exhibits ohmic behavior, indicating higher internal resistance of the ZG MSC compared with the rGO MSC, 
a)

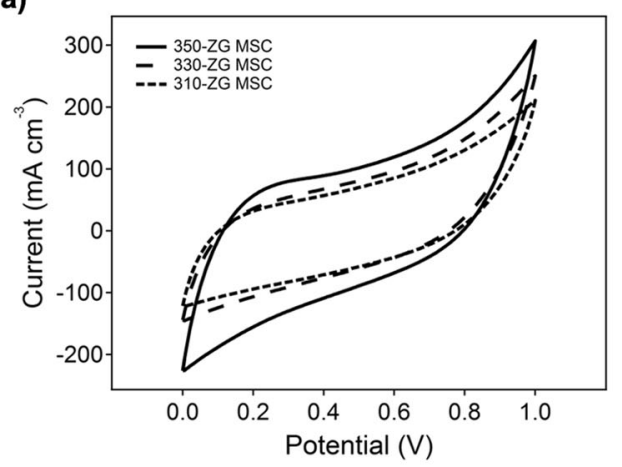

c)

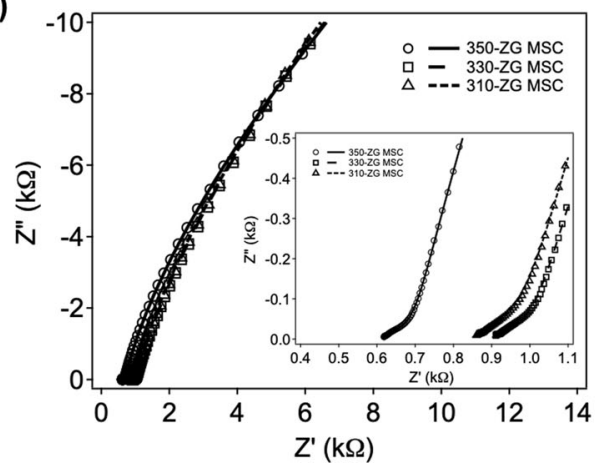

b)

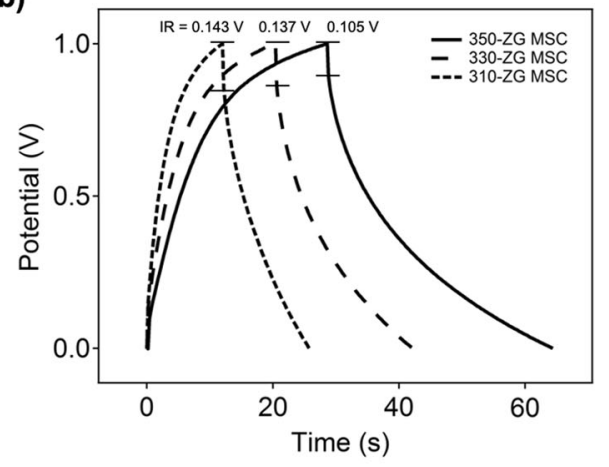

d)

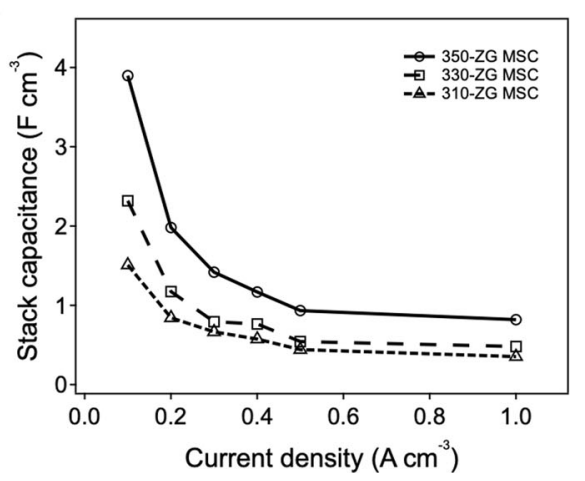

Fig. 4 Electrochemical analyses of the 310-, 330-, and 350-ZG MSCs in the presence of the $\mathrm{H}_{2} \mathrm{SO}_{4} / \mathrm{PVA}$ gel electrolyte. a Cyclic voltammetry curves at a scan rate of $100 \mathrm{mV} \mathrm{s}^{-1}$. b Galvanostatic charge-discharge curves at $0.1 \mathrm{~A} \mathrm{~cm}^{-3}$. c Nyquist plots of the ZG MSCs analyzed over the frequency range of $100 \mathrm{kHz}$ to $0.01 \mathrm{~Hz}$ at the open-circuit potential. Symbols and lines indicate the experimental data and their fittings based on the equivalent circuit model, respectively. $\mathbf{d}$ Stack capacitance measured from the GCD tests at different current densities.

which is a result of the lower electrical conductivity of the $\mathrm{ZnO}$ NRs. Even though the $\mathrm{ZnO}$ NRs exhibit lower electrical conductivity compared with rGO, the Faradaic current generated by the surface reaction between the $\mathrm{ZnO} \mathrm{NRs}$ and the electrolytes increases the stack capacitance. Notably, the ZG MSC exhibits a considerably higher average area in the $\mathrm{CV}$ test, and the GCD curves of the 350-ZG MSC are significantly more prolonged in comparison to the 350-rGO MSC. Consequently, the stack capacitance calculated during the GCD test is higher for the ZG MSC than for the rGO MSC at different current densities (Supplementary Fig. S12c). Furthermore, the long-term stability of the 350-ZG MSC and 350-rGO MSC was also monitored over 10,000 GCD cycles (Supplementary Fig. S12d). During the first 2,000 cycles, both MSCs were unstable and were supposedly undergoing the stabilization process. Subsequently, both MSCs exhibited good and stable retention rates up to 9,000 cycles. Overall, however, the ZG MSC displays significantly better capacitor performance than the rGO MSC. The ZG MSC exhibits a higher stack capacitance $\left(1.31 \mathrm{~F} \mathrm{~cm}^{-3}\right.$ for the rGO MSC vs. $3.90 \mathrm{~F} \mathrm{~cm}^{-3}$ for the ZG MSC), an improved power density $\left(0.26 \mathrm{~W} \mathrm{~cm}^{-3}\right.$ for the rGO MSC vs. $0.40 \mathrm{~W}$ $\mathrm{cm}^{-3}$ for the ZG MSC), and an enhanced energy density, i.e., a higher stack capacitance $\left(0.13 \mathrm{mWh} \mathrm{cm}^{-3}\right.$ for the rGO MSC vs. $0.43 \mathrm{mWh} \mathrm{cm}^{-3}$ for the ZG MSC). The CV curves at different scan rates (from 10 to $500 \mathrm{mV} \mathrm{s}^{-1}$ ) and the GCD curves at various current densities (from 114 to $327 \mathrm{~mA} \mathrm{~cm}^{-3}$ ) were measured for both the rGO MSC and ZG MSC, exhibiting excellent capacitive characteristics and remarkable rate performance of the ZG MSC compared with the rGO MSC (Supplementary Fig. S13). Moreover, the Ragone plots demonstrate that the ZG MSC delivers high power density and energy density, comparable to other supercapacitors with different design structures (Supplementary Fig. S14) ${ }^{1-4,28-30}$. Our patterned rGO, which was reduced utilizing the LightScribe DVD drive, exhibits a lower current level in the IV curve $(800 \mu \mathrm{A}$ at $1 \mathrm{~V})$ than that previously reported by El-Kady et al. ( $20 \mathrm{~mA}$ at $1 \mathrm{~V}$ ) (Supplementary Fig. S15), whereas the current level of GO is analogous to that in the previous work $^{3}$. The different capacitor performances demonstrated in the current study may originate from the different laser power densities and scanning speeds of the LightScribe DVD equipment used in our work than those employed in the previous study by El-Kady et al., significantly affecting the $\mathrm{GO}$ reduction and subsequent electrical conductivity of rGO. It is noteworthy that the 
report on a supercapacitor fabricated using laser-scribed rGO employing a LightScribe DVD recorder identical to the one described in our work demonstrated similar IV characteristics for both $\mathrm{GO}$ and $\mathrm{rGO}^{31}$. Accordingly, a significant performance enhancement can be expected by fabricating our ZG MSCs utilizing the same quality of rGO as described in El-Kady's work ${ }^{3}$. To evaluate the practical applicability of the ZG MSCs, the selfdischarging behavior of the 350-ZG MSC was monitored after charging it to $1 \mathrm{~V}$. Self-discharge is a phenomenon in which the supercapacitor continuously loses stored charges under open-circuit conditions, which is observed as a decrease in the output voltage of the supercapacitor. Since self-discharging is a crucial factor for real applications, great efforts have been made to suppress self-discharge and allow supercapacitors to store energy more efficiently ${ }^{32-35}$. As shown in Fig. S16, the open-circuit potential decreases from $1 \mathrm{~V}$ to $0.6 \mathrm{~V}$ in $30 \mathrm{~min}$, then stabilizes at approximately $0.4 \mathrm{~V}$ and remains at this value for over $15 \mathrm{~h}$. The self-discharge rate can be further suppressed by introducing proper passivation, adding additives, or using organic or solid electrolytes.

\section{Conclusion}

In conclusion, we successfully developed a facile selective patterning method for $\mathrm{ZnO} \mathrm{NR} / \mathrm{rGO}$ composites through a simple photothermal reaction utilizing laser scribing and sequential hydrothermal growth of $\mathrm{ZnO}$. Owing to the design flexibility that the laser-scribing approach provides, various microsupercapacitor designs can be easily examined to determine the optimal device structure. Furthermore, the ZG MSC exhibits notable improvement in all aspects of the capacitor evaluation compared with the rGO MSC, which may be attributed to the synergistic effects of the pseudocapacitance and EDLC of the ZG MSC. In addition, the ZG MSC also exhibits long-term cycling stability. The selective growth of graphene/metal oxide composites, particularly at desired locations, is a challenging but essential task to be adopted for different device applications. We expect that the described technique can be utilized for fabricating various MSCs and functional microdevices by combining the advantages of top-down fabrication and bottom-up synthesis.

\section{Acknowledgements \\ This research was supported by the "Creative Materials Discovery Program" (NRF-2017M3D1A1039379) and "the Basic Research Program" (NRF- 2017R1A2B4007641, NRF-2019R1A2C1010384) through the National Research Foundation of Korea (NRF) funded by the Ministry of Science and ICT. This research was also supported by Samsung Electronics Co., Ltd.}

\section{Author details}

'Department of Applied Chemistry, Kyung Hee University, Yongin, Gyeonggi 17104, Korea. ${ }^{2}$ Department of Materials Science and Engineering, Korea University, Seoul 02841, Korea. ${ }^{3}$ Department of Electrical Engineering, Chungnam National University, Daejeon 34134, Korea

\section{Author contributions}

The manuscript was written through contributions of all authors. All authors have given approval to the final version of the manuscript.

\section{Conflict of interest}

The authors declare that they have no conflicts of interest.

\section{Publisher's note}

Springer Nature remains neutral with regard to jurisdictional claims in published maps and institutional affiliations.

Supplementary information is available for this paper at https:/doi.org/ 10.1038/s41427-020-0230-y.

Received: 7 November 2019 Accepted: 30 April 2020. Published online: 17 July 2020

\section{References}

1. Wu, Z. S., Parvez, K., Feng, X. \& Müllen, K. Graphene-based in-plane microsupercapacitors with high power and energy densities. Nat. Commun. 4, 2487 (2013).

2. Kim, S.-K., Koo, H.-J., Lee, A. \& Braun, P. V. Selective wetting-induced microelectrode patterning for flexible micro-supercapacitors. Adv. Mater. 26, 5108-5112 (2014)

3. El-Kady, M. F. \& Kaner, R. B. Scalable fabrication of high-power graphene micro-supercapacitors for flexible and on-chip energy storage. Nat. Commun. 4, 1475 (2013).

4. Peng, Z Z et al. Flexible boron-doped laser-induced graphene microsupercapacitors. ACS Nano 9, 5868-5875 (2015).

5. Hummers, W. S. \& Offeman, R. E. Preparation of graphitic oxide. J. Am. Chem. Soc. 80, 1339-1339 (1958).

6. Strong, V. et al. Patterning and electronic tuning of laser scribed graphene for flexible all-carbon devices. ACS Nano 6, 1395-1403 (2012).

7. Gilje, S. et al. Photothermal deoxygenation of graphene oxide for patterning and distributed ignition applications. Adv. Mater. 22, 419-423 (2010).

8. Greene, L. E. et al. General route to vertical $\mathrm{ZnO}$ nanowire arrays using textured ZnO seeds. Nano Lett. 5, 1231-1236 (2005).

9. Kwon, J. et al. Direct selective growth of $\mathrm{ZnO}$ nanowire arrays from inkjetprinted zinc acetate precursor on a heated substrate. Nanoscale Res. Lett. 8, 1-6 (2013).

10. Lin, C.-C. \& Li, Y.-Y. Synthesis of $\mathrm{ZnO}$ nanowires by thermal decomposition of zinc acetate dihydrate. Mater. Chem. Phys. 113, 334-337 (2009).

11. Arii, T. \& Kishi, A. The effect of humidity on thermal process of zinc acetate. Thermochim. Acta 400, 175-185 (2003).

12. Greene, L. E. et al. Low-temperature wafer-scale production of $\mathrm{ZnO}$ nanowire arrays. Angew. Chem. Int. Ed. 42, 3031-3034 (2003).

13. Schniepp, H. C. et al. Functionalized single graphene sheets derived from splitting graphite oxide. J. Phys. Chem. B 110, 8535-8539 (2006).

14. $\mathrm{Mu}, \mathrm{X} ., \mathrm{Wu}, \mathrm{X}$., Zhang, T., Go, D. B. \& Luo, T. Thermal transport in graphene oxide - from ballistic extreme to amorphous limit. Sci. Rep. 4, 3909 (2014).

15. Umar, A., Ribeiro, C., Al-Hajy, A., Masuda, Y. \& Hahn, Y. B. Growth of highly Caxis-oriented $\mathrm{ZnO}$ nanorods on $\mathrm{ZnO} /$ glass substrate: growth mechanism, structural, and optical properties. J. Phys. Chem. C. 113, 14715-14720 (2009).

16. Lee, J. E. et al. Catalytic synergy effect of $\mathrm{MoS}_{2} /$ reduced graphene oxide hybrids for a highly efficient hydrogen evolution reaction. RSC Adv. 7, 5480-5487 (2017).

17. Li, M. et al. Facile synthesis of NiAl-layered double hydroxide/graphene hybrid with enhanced electrochemical properties for detection of dopamine. Nanoscale 3, 4240-4246 (2011).

18. Lloyd, J. S., Fung, C. M., Alvim, E. J., Deganello, D. \& Teng, K. S. UV photodecomposition of zinc acetate for the growth of $\mathrm{ZnO}$ nanowires. Nanotechnology 26, 265303 (2015).

19. Mar, L. G., Timbrell, P. Y. \& Lamb, R. N. An XPS study of zinc oxide thin film growth on copper using zinc acetate as a precursor. Thin Solid Films 223, 341-347 (1993).

20. Park, S. et al. Graphene oxide papers modified by divalent ions-enhancing mechanical properties via chemical cross-linking. ACS Nano 2, 572-578 (2008).

21. Yu, Z., Tetard, L., Zhai, L. \& Thomas, J. Supercapacitor electrode materials: nanostructures from 0 to 3 dimensions. Energy Environ. Sci. 8, 702-730 (2015). 
22. Rakhi, R. B., Alhebshi, N. A., Anjum, D. H. \& Alshareef, H. N. Nanostructured cobalt sulfide-on-fiber with tunable morphology as electrodes for asymmetric hybrid supercapacitors. J. Mater. Chem. A 2, 16190-16198 (2014).

23. Yun, J. et al. A patterned graphene/ZnO UV sensor driven by integrated asymmetric micro-supercapacitors on a liquid metal patterned foldable paper Adv. Funct. Mater. 27, 1700135 (2017).

24. Qu, Z. et al. An efficient binder-free electrode with multiple carbonized channels wrapped by $\mathrm{NiCO}_{2} \mathrm{O}_{4}$ nanosheets for high-performance capacitive energy storage. J. Power Sources 410-411, 179-187 (2019).

25. Ghosh, A. \& Lee, Y. H. Carbon-based electrochemical capacitors. ChemSusChem 5, 480-499 (2012).

26. Okafor, P. A. \& Iroh, J. O. Fabrication of porous graphene/polyimide composites using leachable poly-acrylic resin for enhanced electrochemical and energy storage capabilities. J. Mater. Chem. A 3, 17230-17240 (2015).

27. Zhang, S. \& Pan, N. Supercapacitors performance evaluation. Adv. Energy Mater. 5, 1401401 (2015).

28. El-Kady, M. F., Strong, V., Dubin, S. \& Kaner, R. B. Laser scribing of highperformance and flexible graphene-based electrochemical capacitors. Science 335, 1326-1330 (2012).
29. Lin, J. et al. 3-Dimensional graphene carbon nanotube carpet-based microsupercapacitors with high electrochemical performance. Nano Lett. 13, 72-78 (2013).

30. Jung, H. Y., Karimi, M. B., Hahm, M. G., Ajayan, P. M. \& Jung, Y. J. Transparent, flexible supercapacitors from nano-engineered carbon films. Sci. Rep. 2, 773 (2012).

31. Tian, $H$. et al. Scalable fabrication of high-performance and flexible graphene strain sensors. Nanoscale 6, 699-705 (2014).

32. Shi, M., Xiao, P., Lang, J., Yan, C. \& Yan, X. Porous $g-\mathrm{C}_{3} \mathrm{~N}_{4}$ and MXene dualconfined $\mathrm{FeOOH}$ quantum dots for superior energy storage in an ionic liquid. Adv. Sci. 7, 1901975 (2020)

33. Wang, Z. et al. Extremely low self-discharge solid-state supercapacitors via the confinement effect of ion transfer. J. Mater. Chem. A 7, 8633-8640 (2019).

34. Liu, Y. et al. High-performance coaxial wire-shaped supercapacitors using ionogel electrolyte toward sustainable energy system. J. Mater. Res. 34, 3030-3039 (2019).

35. Shi, M. et al. Boosting ion dynamics through superwettable leaf-like film based on porous $\mathrm{g}-\mathrm{C}_{3} \mathrm{~N}_{4}$ nanosheets for ionogel supercapacitors. NPG Asia. Materials 11, 61 (2019). 